\title{
Prediction of Skin Permeation of Flurbiprofen from Neat Ester Oils and Their $O / W$ Emulsions
}

\author{
Hiroaki Todo ${ }^{a, b}$ Mai Tamura, ${ }^{a}$ Takashi Uchida ${ }^{c}$ Miyuki Kurumada, ${ }^{a}$ Anzu Motoki, ${ }^{a}$ \\ Tomohiko Sano, ${ }^{d}$ Yoshinori Onuki, ${ }^{e}$ Kozo Takayama, ${ }^{b}$ and Kenji Sugibayashi*,a,b \\ ${ }^{a}$ Graduate School of Pharmaceutical Sciences, Josai University; 1-1 Keyakidai, Sakado, Saitama 350-0295, Japan: \\ ${ }^{b}$ Faculty of Pharmacy and Pharmaceutical Sciences, Josai University; 1-1 Keyakidai, Sakado, Saitama 350-0295, \\ Japan: ${ }^{c}$ R\&D-Development Research, Kao Corporation; 5-3-28 Bunka, Sumida-ku, Tokyo 131-8502, Japan: \\ ${ }^{d}$ Faculty of Life and Environmental Sciences, Teikyo University of Science; 2-2-1 Senju Sakuragi, Adachi-ku, Tokyo \\ 120-0045, Japan: and ${ }^{e}$ Department of Pharmaceutical Technology, Graduate School of Medical and Pharmaceutical \\ Science, University of Toyama; 2630 Sugitani, Toyama 930-0194, Japan.
}

Received May 20, 2018; accepted July 19, 2018

\begin{abstract}
Although many in silico models were reported to predict the skin permeation of drugs from aqueous solutions, few studies were founded on the in silico estimation models for the skin permeation of drugs from neat oil formulations and $o / w$ emulsions. In the present study, the cumulative amount of a model lipophilic drug, flurbiprofen (FP), that permeated through skin was determined from 12 different kinds of ester oils $\left(Q_{o i l}\right)$ and an in silico model was developed for predicting the skin permeation of FP from these ester oils. Thus, the obtained $Q_{o i l}$ values were well predicted with the FP solubility in the oils $\left(S_{o i l}\right)$, the amount of FP uptake into the stratum corneum $\left(S C_{o i l}\right)$ and molecular descriptors of dipolarity/polarizability $\left(\pi_{2}{ }^{H}\right)$ and molecular density. This model suggests that the thermodynamic activities of FP both in the formulations and skin are the key factors for predicting the skin permeation of FP from the ester oils. In addition, a high linear relationship was observed in the double-logarithm plots between the $Q_{o i l}$ and the cumulative amount of FP permeated through skin from $20 \%$ ester oil in water emulsion $\left(Q_{\text {emul20\% }}\right)$. Furthermore, the skin permeations of FP from 5 and $10 \%$ ester oil in water emulsions, $Q_{\text {emuls\% }}$ and $Q_{\text {emul10\%, respectively, were also }}$ predicted by the horizontal translation of the $y$-axis intercept of the liner equation for the relation between the $Q_{o i l}$ and $Q_{\text {emul20\% }}$. These prediction methods must be helpful for designing topical oily and/or $o / w$ emulsion formulations having suitable and high skin permeation rate of lipophilic drugs.
\end{abstract}

Key words in silico prediction; skin permeation; ester oil; oil in water emulsion; Abraham descriptor

Many in vitro and in vivo skin permeation studies have been conducted to develop topically applied pharmaceutical formulation from the viewpoints of efficacy and safety. However, further development of new topical formulations is prevented by the low availability of excised human skins for ethical reasons and the ban on animal testing in the EU for cosmetic products. Then, establishment of a good in silico skin permeation-estimation model was expected ${ }^{1-5)}$ to overcome these problems. At the same time, development of good three-dimensional cultured human skin models ${ }^{6,7)}$ and synthetic membranes ${ }^{8-10)}$ are expected for alternative skin membranes suitable for the permeation tests. Several in silico skin permeation-estimation models have already published to predict skin permeation of drugs from aqueous solutions: most of them used lipophilicity and molecular size of drugs and based on the skin permeation data set of many drugs from aqueous solutions. The best known one is the 'Potts and Guy' equation. ${ }^{1)}$ However, no or little in silico methods were found on the formulation effects except aqueous formulations on the skin permeation of drugs.

Oil based formulations and oil-in-water $(o / w)$ emulsions are widely used in cosmetic and dermatological formulations. Grégoire et $a l^{5)}$ reported that chemical absorption into and through the skin from $o / w$ emulsions was successfully predicted with $n$-octanol/water coefficient of topically applied chemicals in the formulations. However, several assumptions were necessary for their model, including that only a fraction of the chemical in the continuous (aqueous) phase of the emulsion was available for partitioning into the skin, which must be too hard to realistic assumption.

Skin permeation of drugs can be generally explained by the drug partitioning from the formulation into the uppermost layer of skin, stratum corneum and drug diffusivity in the biggest barrier of skin, stratum corneum. ${ }^{2)}$ Since the barrier function and lipophilicity of the stratum corneum are modified by penetration of the topically applied oil- and water-based components (mostly oil-based components due to much higher uptake into the stratum corneum than water-based components), the effect of various vehicles should be considered in the development of an in silico model to predict skin permeation of drugs from such complex formulations. Sloan et al. investigated the effect of vehicles on the diffusion of salicylic acid through the $\operatorname{skin}^{11)}$ and Sakata et al. reported the dependence of physicochemical properties of the oil vehicles on the skin penetration of compounds. ${ }^{12)}$

Thus, selection of oil vehicles is of great importance in preparing dermatological formulations intended for the improvement of texture and sensory properties. Furthermore, different oil vehicles in the formulation may be used not only to alter the thermodynamic activity of drugs, but also affect the barrier function of the stratum corneum by delipidization, fluidization and disruption. ${ }^{13)}$ Thus, understanding the factors modulating the skin permeation of drugs from oil vehicles is very important in conducting formulation design and develop- 
Table 1. Saturated Solubility of FP in the Ester Oils and Surface Tension of the Ester Oils

\begin{tabular}{lcc}
\hline \hline \multicolumn{1}{c}{ Oil name (Abbre.) } & $\begin{array}{c}\text { Saturated } \\
\text { solubility }\left(S_{\text {oil }}\right) \\
(\mathrm{mg} / \mathrm{mL})\end{array}$ & $\begin{array}{c}\text { Surface tension } \\
(\mathrm{ST}) \\
(\mathrm{mN} / \mathrm{m})\end{array}$ \\
\hline 2-Ethylhexyl isononanoate (EHIN) & 38.6 & 25.8 \\
2-Ethylhexyl palmitate (EHP) & 23.9 & 29.3 \\
Diisopropyl adipate (DIPA) & 135 & 28.3 \\
Di-2-ethylhexyl sebacate (DEHS) & 58.5 & 30.6 \\
Propylene glycol isostearate (PGIS) & 77.7 & 30.6 \\
Diethyl sebacate (DES) & 161 & 32.2 \\
2-Octyldodecyl ricinoleate (ODR) & 65.0 & 31.7 \\
2-Hexyldecyl isostearate (HDIS) & 11.3 & 29.6 \\
Isononyl isononanoate (ININ) & 31.9 & 25.5 \\
Neopentyl glycol di(2-ethylhexanoate) & 52.3 & 28.0 \\
$\quad$ (NPGDEH) & & \\
Isotridecyl isononanoate (ITDIN) & 23.3 & 27.2 \\
Dioctyl carbonate (DOC) & 37.4 & 28.8 \\
\hline
\end{tabular}

ing a prediction model for skin permeation of drugs.

In the present study, 12 ester oils, as shown in Table 1, were selected and the skin permeation of a lipophilic drug, flurbiprofen (FP), from the ester oils was investigated by an in vitro skin permeation experiment. Flurbiprofen was selected as a model drug due to its lipophilicity and high skin permeation. Multi-regression analysis was implemented to establish an in silico model for predicting the skin permeation of FP from the ester oils. Furthermore, the relationship was evaluated between FP permeation from ester oils and that from $o / w$ emulsions. In addition, a prediction model for FP permeation from different ester oil contents in water emulsions was established by considering both the obtained in silico model and the relationship in skin permeation of FP from ester oils and $o / w$ emulsions.

\section{Experimental}

Materials FP was kindly provided by Lead Chemical Co., Ltd. (Toyama, Japan). Nine ester oils; diisopropyl adipate (DIPA), isononyl isononanoate (ININ), neopentyl glycol di(2ethylhexanoate) (NPGDEH), 2-ethylhexyl palmitate (EHP), di-2-ethylhexyl sebacate (DEHS), dioctyl carbonate (DOC), isotridecyl isononanoate (ITDIN), propylene glycol isostearate (PGIS) and 2-octyldodecyl ricinoleate (ODR) were supplied by the Kao Corporation (Tokyo, Japan). The other 3 ester oils; diethyl sebacate (DES), 2-ethylhexyl isononanoate (EHIN) and 2-hexyldecyl isostearate (HDIS) were given by Nikko Chemicals Co., Ltd. (Tokyo, Japan). Table 1 summarizes them. Acrylates/C10-30 alkyl acrylate crosspolymer (Lubrizol Corporation, Ohio, U.S.A.) was provided by Kao Corporation. Frozen edible pig ear skins (17-23 weeks of age) were purchased from the National Federation of Agricultural Cooperative Associations (Tokyo, Japan). These skin pieces were stored at $-80^{\circ} \mathrm{C}$ until the in vitro experiments. All other reagents and solvents were of reagent grade or HPLC grade and used without further purification.

Saturated Solubility of FP An excess amount of FP was added into purified water or each ester oil to obtain a suspension. The FP suspension was stirred at $32^{\circ} \mathrm{C}$ for $48 \mathrm{~h}$, and the FP-saturated solution was obtained by passing the suspension through a filter (Millex-LH $0.45 \mu \mathrm{m}$ or DISMIC ${ }^{\circledR}-13 \mathrm{HP}$ $0.45 \mu \mathrm{m}$; Advantec Toyo Kaisha, Ltd., Tokyo, Japan). The
Table 2. Composition of $O / W$ Emulsions

\begin{tabular}{|c|c|c|c|c|}
\hline \multirow[b]{2}{*}{ Component } & \multicolumn{4}{|c|}{ Ester oil conc. } \\
\hline & $5 \%$ & $10 \%$ & $20 \%$ & $30 \%$ \\
\hline FP & \multicolumn{4}{|c|}{0.2} \\
\hline Ester oil & 1 & 2 & 4 & 6 \\
\hline Purified water & 17.13 & 16.13 & 14.13 & 12.13 \\
\hline $\begin{array}{l}2.0 \% \text { acrylates } / \mathrm{C} 10-30 \text { alkyl acrylate } \\
\text { crosspolymer gel }\end{array}$ & \multicolumn{4}{|c|}{1.6} \\
\hline $20 \% \mathrm{KOH}$ & \multicolumn{4}{|c|}{0.05} \\
\hline Phenoxy ethanol & \multicolumn{4}{|c|}{0.02} \\
\hline Total & \multicolumn{4}{|c|}{20} \\
\hline
\end{tabular}

Unit (g).

obtained saturated solution was diluted with ethanol, and then the FP solubility in water and each ester oil, $S_{\text {water }}$ and $S_{\text {oil }}$, respectively, was determined by HPLC.

Preparation of $\boldsymbol{O} / \boldsymbol{W}$ Emulsions Twenty percent oil in water emulsions were prepared using each of 12 ester oils by the following procedure: First, 2.0\% acrylates/C10-30 alkyl acrylate crosspolymer gel was prepared by mixing with purified water by Labolution ${ }^{\circledR}$ (PRIMIX Corporation, Tokyo, Japan) at $500 \mathrm{rpm}$ at $32^{\circ} \mathrm{C}$ for $5 \mathrm{~min}$. Then, $1.6 \mathrm{~g}$ of $2.0 \%$ acrylates/C10-30 alkyl acrylate crosspolymer gel, $14.13 \mathrm{~g}$ of purified water, $4.0 \mathrm{~g}$ of ester oil and $0.2 \mathrm{~g}$ of FP were well mixed by Labolution ${ }^{\circledR}$ at $4500 \mathrm{rpm}$ at $80^{\circ} \mathrm{C}$ for $3 \mathrm{~min}$. FP was completely dissolved in the ester oil before mixing. After $0.05 \mathrm{~g}$ of $20 \%$ potassium hydroxide solution was added the solution, the obtained solution was mixed by Labolution ${ }^{\circledR}$ at $4500 \mathrm{rpm}$ at $70^{\circ} \mathrm{C}$ for $5 \mathrm{~min}$. Phenoxy ethanol $(0.02 \mathrm{~g})$ was then mixed by Labolution ${ }^{\circledR}$ at $4500 \mathrm{rpm}$ at $40^{\circ} \mathrm{C}$ for $3 \mathrm{~min}$ to finally obtain $20 \mathrm{~g}$ of $o / w$ emulsion containing FP at a concentration of $1.0 \%$. In addition to this $20 \%$ ester oil in water emulsion, 5, 10 and $30 \%$ of ester oil in water emulsions were prepared by changing the amount of water and ester oil in the formulation. Furthermore, aqueous acrylates/C10-30 alkyl acrylate crosspolymer gel was used without adding ester oil in the $o / w$ emulsion preparation process. Table 2 summarizes these formulations.

Solvent Uptake Skin tissue was excised from the dorsal auricular, then the stratum corneum (SC) sheet was made by trypsin treatment. ${ }^{14)}$ Then, the obtained sheet was cut into $1.0 \times 1.0 \mathrm{~cm}^{2}$ size. After weighing the $1.0 \times 1.0 \mathrm{~cm}^{2}$ sheet by a microbalance (Shimadzu Corporation, Kyoto, Japan), it was stored at a vapor-saturated condition using saturated $\mathrm{NaCl}$ solution in water at $25 \pm 5^{\circ} \mathrm{C}(75 \pm 5 \% \mathrm{RH})$ for $24 \mathrm{~h}^{15)}$ Then, the water hydrated SC sheet was again weighed. The hydrated $\mathrm{SC}$ sheet was immersed into $3.0 \mathrm{~mL}$ of each ester oil, aqueous gel or prepared $o / w$ emulsion at $32^{\circ} \mathrm{C}$ for $1 \mathrm{~h}$. Then, the SC sheet was removed from the solvent, blotted on Kim Towel ${ }^{\circledR}$ (Nippon Paper Crecia Co., Ltd., Tokyo, Japan) with loading a weight of $1.5 \mathrm{~kg}\left(18.5 \times 13 \mathrm{~cm}^{2}\right)$ for $10 \mathrm{~s}$, and weighed again. The relative mass uptake of each ester oil or $o / w$ emulsion per gram of the sheet was calculated from the difference between the weights of the SC sheet before and after soaking.

In Vitro Skin Permeation Experiment with Ester Oil In vitro skin permeation experiments were performed using a diffusion cell array system (Ikeda Scientific Co., Ltd., Tokyo, Japan). This system was previously reported in detail, ${ }^{16)}$ and used to reduce the skin area to conduct skin permeation experiment.

Excised pig ear skin $(8.5 \times 12.5 \mathrm{~cm})$ after removal of subcuta- 
neous adipose tissue was set in the diffusion cell array system with an effective permeation area of $0.785 \mathrm{~cm}^{2}$ to determine the cumulative amounts of FP through the skin from each ester oil over $5 \mathrm{~h}\left(Q_{o i}\right)$. FP solution $(10 \mathrm{mg} / \mathrm{g})$ was prepared by completely dissolving $\mathrm{FP}$ with ester oil at $80^{\circ} \mathrm{C}$ followed by stirring at $32^{\circ} \mathrm{C}$ for $48 \mathrm{~h}$. The FP in ester oil $(1.0 \mathrm{~mL})$ was added to the donor cell, whereas phosphate-buffered saline (PBS; pH 7.4) was used as a receiver solution $(1.4 \mathrm{~mL})$. Each solution was stirred using a stir ball and maintained at $32^{\circ} \mathrm{C}$ using a thermo-shaker. The receiver solutions were then collected $5 \mathrm{~h}$ after starting the experiment. The FP concentration in the receiver solution was assayed using HPLC to determine $Q_{\text {oil }}$ value.

In Vitro Skin Permeation Experiment with $O / W$ Emulsion Excised pig ear skin was mounted on vertical-type diffusion cells (effective diffusion area: $1.77 \mathrm{~cm}^{2}$ ). After hydrated the SC with PBS for $1 \mathrm{~h}$, the PBS was completely removed from the diffusion cell and $1.0 \mathrm{~mL}$ of $o / w$ emulsion containing FP and $6 \mathrm{~mL}$ of PBS were applied to the donor and receiver cells, respectively. The permeation experiments were performed at $37^{\circ} \mathrm{C}$ by the use of circulating water to maintain skin surface temperature $32^{\circ} \mathrm{C}$, while the receiver solution was continuously stirred with a star-head-type magnetic stirrer. At predetermined times, an aliquot $(0.5 \mathrm{~mL})$ was withdrawn from the receiver solution and the same volume of fresh solution was added to keep the volume constant to follow the time course of skin permeation of FP over 5h. Each experiment was performed for $5 \mathrm{~h}$ with three to four replications. The concentration of FP in the receiver solution was assayed using HPLC to determine the cumulative amount of FP that permeated from $o / w$ emulsion through the skin $\left(Q_{\text {emul }}\right)$.

HPLC Analysis FP concentrations in the samples were determined using an HPLC system (Prominence; Shimadzu) equipped with a UV detector (SPD-M20A; Shimadzu). The drug samples were centrifuged at $21500 \times \boldsymbol{g}$ and $4^{\circ} \mathrm{C}$ for $5 \mathrm{~min}$, $20 \mu \mathrm{L}$ of the supernatant was added to the same volume of acetonitrile, and the mixed solution was injected into the HPLC system. Chromatographic separation was performed using an Inertsil-ODS-3 $\left(5 \mu \mathrm{m}, 150 \times 4.6 \mathrm{~mm}^{2}\right.$ i.d.; GL Science, Kyoto, Japan) at $40^{\circ} \mathrm{C}$. The mobile phase was $0.1 \%$ phosphoric acid/acetonitrile $(50 / 50, \mathrm{v} / \mathrm{v})$. The flow rate was adjusted to $1.0 \mathrm{~mL} / \mathrm{min}$, and detection was performed at UV $254 \mathrm{~nm}$. The obtained standard calibration curve was prepared from a concentration of 1.0 to $100 \mu \mathrm{g} / \mathrm{mL}$ (the lower limit of quantification was $0.1 \mu \mathrm{g} / \mathrm{mL})$.

Surface Tension (ST) of the Ester Oils The ST of the ester oils was determined using a Dropmaster DM-701 (Kyowa Interface Science, Niiza, Saitama, Japan). ST was measured using the pendant drop method at $25^{\circ} \mathrm{C}$ with six replicates.

Quantitative Structure-Property Relationship (QSPR) Modeling Approach Multi-regression analysis was carried out using $\mathrm{JMP}^{\circledR}$ Pro (ver. 13.1.0, SAS Institute, Cary, NC, U.S.A.). Stepwise regression analysis was employed to develop a regression, and the most suitable descriptors were selected using coefficient of variation $\left(r^{2}\right)$. Separately, stepwise regression was used to select the best factors to predict $Q_{\text {oil }}$ or $Q_{\text {emul }}$ values, based on the $r^{2}$ value, from descriptors retrieved from the ACD/Dictionary and measured parameters. ACDLabs software package (ACD/Percepta ver. 14.0.0) was used to calculate the molecular descriptors for ester oils. The following descriptors were selected in the present study (see in detail in Table 3); molecular weight (M.W.), molecular density $(d)$, excessive molar refraction $\left(M_{\text {ref }}\right)$, number of hydrogen bond acceptors (HA), topological polar surface area (tPSA), freely rotatable bonds $(F R B)$, index of refraction $(I R)$, hetero ratio, $\mathrm{C}$ ratio, $\mathrm{N}$ ratio, nitric oxide $(\mathrm{NO})$ ratio, Parachor $(\mathrm{Pa})$, dipolarity/po-

Table 3. Molecular Descriptors of Oils and Measured Parameters Used in This Experiment

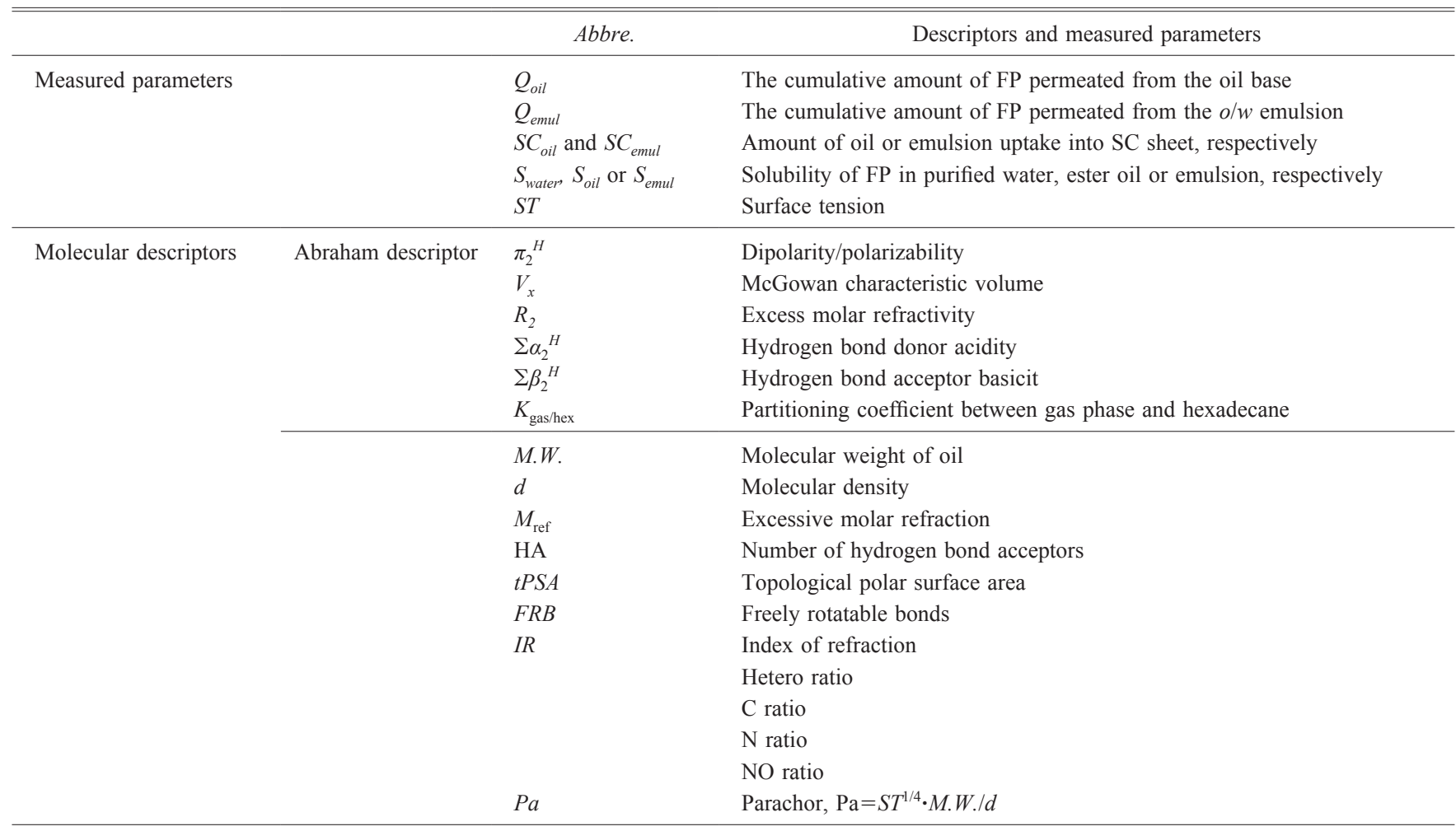




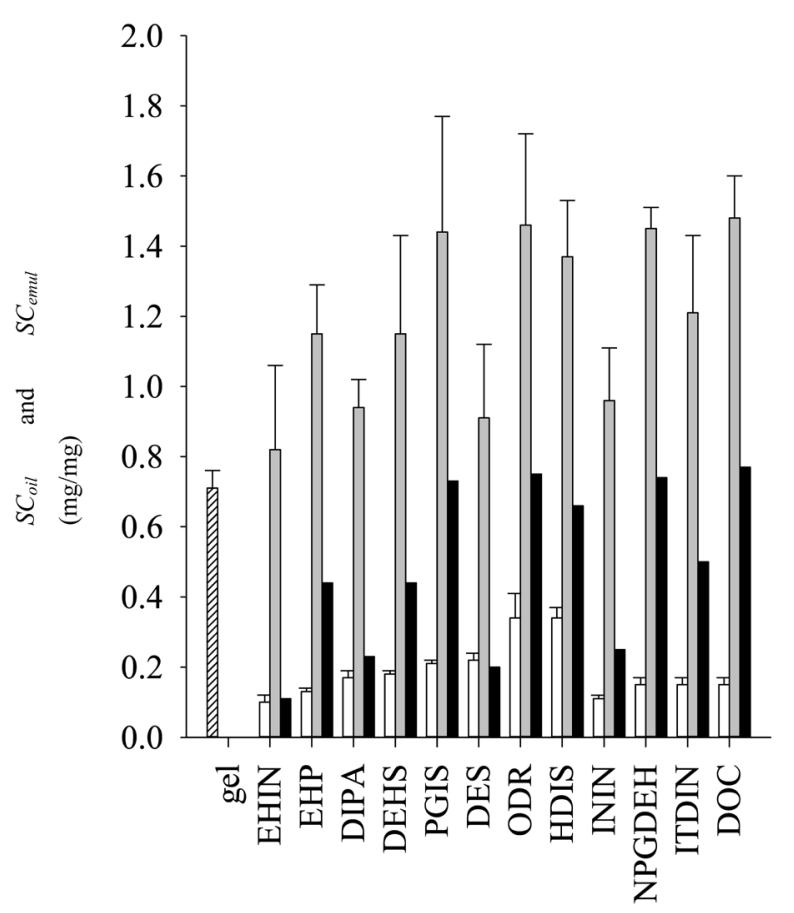

Fig. 1. Amount of Ester Oil Uptake into the Stratum Corneum

Open bar; amount of ester oil uptake, gray bar; amount of $o / w$ emulsion uptake, closed bar; calculated amount of ester oil uptake by subtraction of the aqueous gel uptake amount from that of $o / w$ emulsion uptake, hatched bar; amount of aqueous gel uptake. Mean \pm standard deviation (S.D.) $(n=3)$.

larizability $\left(\pi_{2}^{H}\right)$, McGowan characteristic volume $\left(V_{x}\right)$, excess molar refractivity $\left(R_{2}\right)$, hydrogen bond donor acidity $\left(\Sigma \alpha_{2}^{H}\right)$, hydrogen bond acceptor basicity $\left(\Sigma \beta_{2}^{H}\right)$ and Partitioning coefficient between gas phase and hexadecane $\left(K_{\text {gas/hex }}\right)$. In addition, amount of oil or emulsion uptake into the SC sheet $\left(S C_{o i l}\right.$ and $S C_{\text {emul }}$, respectively), solubility of FP in purified water, ester oil or emulsion $\left(S_{\text {water }}, S_{\text {oil }}\right.$ and $S_{\text {emul }}$, respectively) and $S T$. Leave-some-25\%-out cross-validation was applied to predict the correlation accuracy of the equation. The descriptors $\pi_{2}^{H}$, $V_{x}, R_{2}, \Sigma \alpha_{2}^{H}, \Sigma \beta_{2}^{H}$ and $K_{\text {gas/hex }}$ are known as Abraham descriptors, ${ }^{17)}$ which have been widely used to predict partition coefficient of drugs into blood and body organs. ${ }^{18)}$

\section{Results}

Solubility of FP in the Ester Oils Table 1 shows solubility of FP in each oil $\left(S_{o i l}\right)$ for each ester oil. Lower $S_{\text {oil }}$ was observed in ester oils such as ITDIN, EHP, and HDIS, whereas DIPA and DES and PGIS displayed higher $S_{\text {oil }}$. The Surface tension (ST) values of oils used in the present study were 25.5 to $32.2 \mathrm{mN} / \mathrm{m}$.

Solvent Uptake into the Stratum Corneum from Ester Oil or Emulsion The $S C_{\text {oil }}$ value is summarized in Fig. 1. A large difference in the amount of solvent uptake was observed. Low $S C_{\text {oil }}$ was observed in EHIN $(0.098 \mathrm{mg} / \mathrm{g})$ and ININ $(0.11 \mathrm{mg} / \mathrm{g})$ and high $S C_{\text {oil }}$ was for HDIS $(0.34 \mathrm{mg} / \mathrm{g})$ and ODR $(0.34 \mathrm{mg} / \mathrm{g})$. No relationship was confirmed between $S C_{o i l}$ and $S_{\text {sol }}\left(r=0.23\right.$, data not shown). In addition, $S C_{\text {emul }}$ was also investigated. Since $o / w$ emulsion was composed of ester oil and aqueous gel, the amount of aqueous gel uptake into the SC was also measured for comparison. The amount of the ester oil uptake into the SC sheet after application of $o / w$ emulsion was calculated by the difference between the $S C_{\text {emul }}$ and the amount of aqueous gel into the SC sheet. In almost all cases,

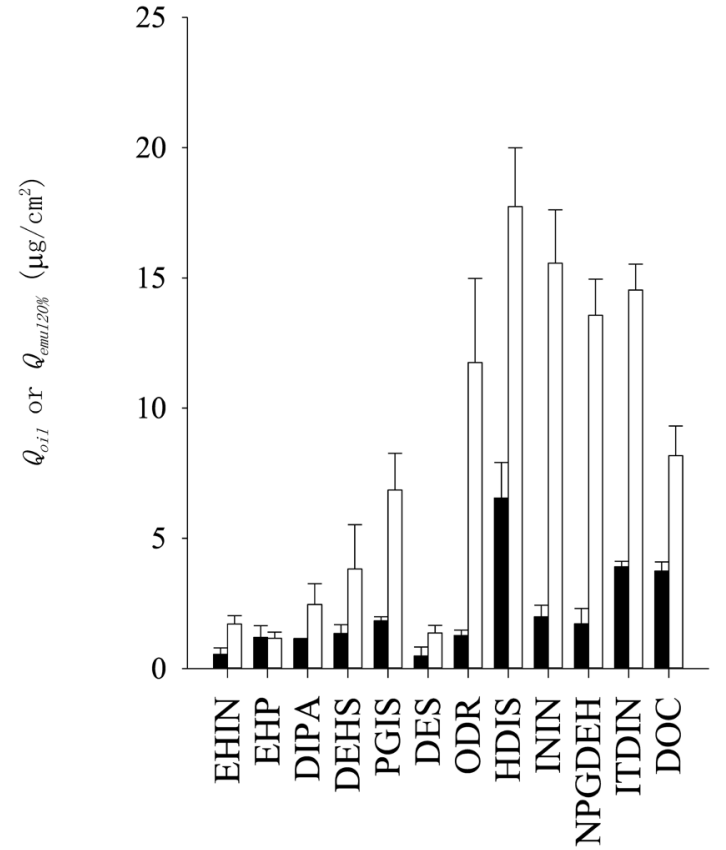

Fig. 2. Cumulative Amount of FP Permeated through the Skin from Ester Oil $\left(Q_{\text {oil }}\right)$ and $O / W$ Emulsion Containing $20 \%$ Ester Oil $\left(Q_{\text {emul20\% }}\right)$ over $5 \mathrm{~h}$

Closed bar; $Q_{\text {oil }}$ value, Open bar; $Q_{\text {emul20\% }}$ value. Mean \pm standard error (S.E.) $(n=4-5)$.

the amounts of oil uptake into the SC sheet were increased by the application of $o / w$ emulsion compared with neat ester oils, although the calculated oil uptake from the emulsions containing EHIN and DES were near to their $S C_{\text {oil }}$ values.

FP Permeation through Skin Figure 2 shows the $Q_{\text {oil }}$ values over $5 \mathrm{~h}$ obtained from the in vitro skin permeation experiment from neat ester oils. Higher $Q_{\text {oil }}$ values were observed in HDIS, followed by ITDIN and DOC. In contrast, low $Q_{\text {oil }}$ values were observed in EHIN and DES. Figure 3 shows a color map for strong and weak correlations between each parameter of the molecular descriptors (Table 3) and observed values including $Q_{\text {oil }}$. The bright red and blue colors denote positive and negative strong correlations, respectively. Several parameters, such as that between $\pi_{2}^{H}$ and $\Sigma \beta_{2}^{H}$, show a strong negative correlation. However, no good relationship was observed between the $Q_{\text {oil }}$ and the other parameters.

Figure 2 also shows the cumulative amount of FP permeated through pig ear skin from $o / w$ emulsions containing $20 \%$ ester oil in water emulsions $\left(Q_{\text {emul20\%}}\right)$ over $5 \mathrm{~h}$. Higher FP permeations were observed in all $o / w$ emulsions $\left(Q_{\text {emul20\% }}\right.$ than neat oils, $Q_{\text {oil }}$ ). High $Q_{\text {emul } 20 \%}$ values were exhibited by HDIS and ITDIN in water emulsions, whereas $Q_{\text {emul } 20 \%}$ values were low in EHIN and DES emulsions. All emulsions, except EHP emulsion, showed higher FP permeation compared with that from the corresponding neat oils. Although the permeation order of $Q_{\text {emul20\% }}$ did not exactly match to that of the $Q_{\text {oil }}$, a good relationship was confirmed between the double-logarithm plots of $Q_{\text {oil }}$ and $Q_{\text {emul20\% }}$ values, as shown in Fig. 4 . The relationship was expressed by,

$$
\log Q_{\text {emul20\% }}=1.04 \times \log Q_{\text {oil }}+0.52 \quad\left(r^{2}=0.61\right)
$$

Skin Permeation of FP from $o / w$ Emulsions Having Different Concentrations of Ester Oil PGIS, DES and ODR 


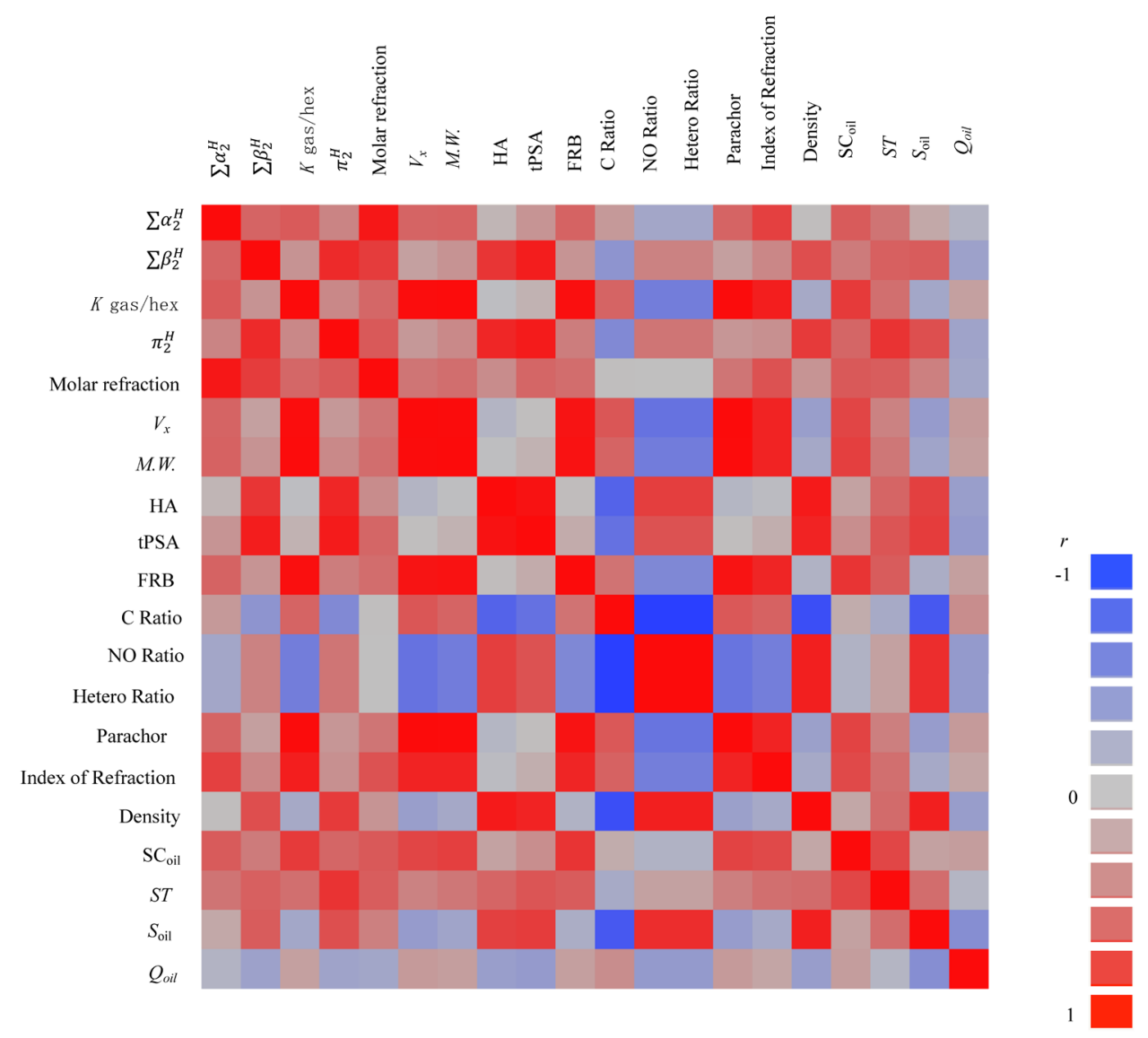

Fig. 3. Colored Map on Correlations between Each Parameter

Bright red indicates a strong correlation, bright blue indicates a strong negative correlation, and gray shows no relationship. Every parameter was perfectly correlated with itself (Shown in the diagonal line from the top left to the bottom right). (Color figure can be accessed in the online version.)

were selected to prepare 5 and $10 \%$ ester oil in water emulsions (shown as PGIS-5, DES-5 and ODR-5 for 5\% emulsions, respectively, and PGIS-10, DES-10 and ODR-10 for 10\% emulsions, respectively) because of the stability of prepared emulsions with different oil concentration. Additionally, 30\% PGIS in water emulsion was prepared (shown as PGIS-30). Figure 5 shows the FP permeation profiles from the $o / w$ emulsions having different concentrations of ester oils. In all formulations, 5\% ester oil in water emulsions showed the highest permeation compared with other $o / w$ formulations and FP permeation was decreased with an increase in the ester oil \% in the formulation. When plotted the logarithms of the $Q_{\text {emul }}$ $\left(Q_{\text {emul } 5 \%}, Q_{\text {emull10\% }}, Q_{\text {emul } 20 \%}, Q_{\text {emul } 30 \%}\right)$ and $Q_{\text {oil }}$ values against the logarithm of ester oil concentration in the formulation (Fig. 4), linear lines were obtained with similar slopes for each ester oil (PGIS, DES and ODR). The slope (mean \pm standard deviation (S.D.)) obtained by the least squared method from these three ester oils was $-1.01( \pm 0.33)$. In addition, when applied the least-square equation to $Q_{\text {emul } 5 \%}$ and $Q_{\text {emullo\% }}$ values for each emulsion having the same ester oil concentration, as shown in Fig. 4, the following equations were obtained for 5\% and $10 \%$ emulsion, respectively,

$$
\begin{aligned}
& \log Q_{\text {emul } 5 \%}=0.97 \times \log Q_{\text {oil }}+1.25 \quad\left(r^{2}=0.80\right) \\
& \log Q_{\text {emul } 10 \%}=1.11 \times \log Q_{\text {oil }}+0.97 \quad\left(r^{2}=0.91\right)
\end{aligned}
$$

In Silico Estimation of Skin Permeation of FP Multiregression analysis was applied to the $Q_{\text {oil }}$ value. Regression models were optimized using a stepwise variable forward selection method, which gave the smallest values for Bayesian Information Criterion. The optimized equation to predict the $Q_{o i l}$ value is as follows:

$$
\begin{aligned}
& Q_{\text {oil }}=-10.3( \pm 2.7) \pi_{2}^{H}+81.8( \pm 20.9) d+22.9( \pm 2.5) S C_{\text {oil }} \\
& \quad-0.07( \pm 0.02) S_{\text {sol }}-63.5( \pm 17.0) \\
& n=69, R^{2}=0.68, F_{0}(4,64)=30.1,(p<0.001)
\end{aligned}
$$

where $n$ is the number of samples, $R^{2}$ is the coefficient of determination, $F_{0}$ is the observed variance ratio, and the number in the parentheses is the $95 \%$ confidence interval of regression coefficient. In the optimal regression equation for $Q_{o i l}, Q_{o i l}$ was increased with an increase of density and $S C_{\text {oil }}$, and was decreased with an increase of $\pi_{2}^{H}$ and $S_{\text {oil }}$. Figure 6 shows 4-fold cross-validation results (trial \#4) in the prediction model for $Q_{\text {oil }}$. The $R_{C V}^{2}$ value denotes $R^{2}$ for each cross-validation result with $Q_{o i l}$ data ( $75 \%$ training and $25 \%$ validation). The $R_{C V}^{2}$ value in the prediction model for $Q_{o i l}$ was $0.66 \pm 0.05$ (the mean \pm S.D.).

\section{Discussion}

Many reports have shown an in silico model that predicts the permeation coefficient $(P)$ of chemical compounds through skin from water-based formulation. Potts and Guy developed a widely accepted in silico prediction model for the $\log p$ 


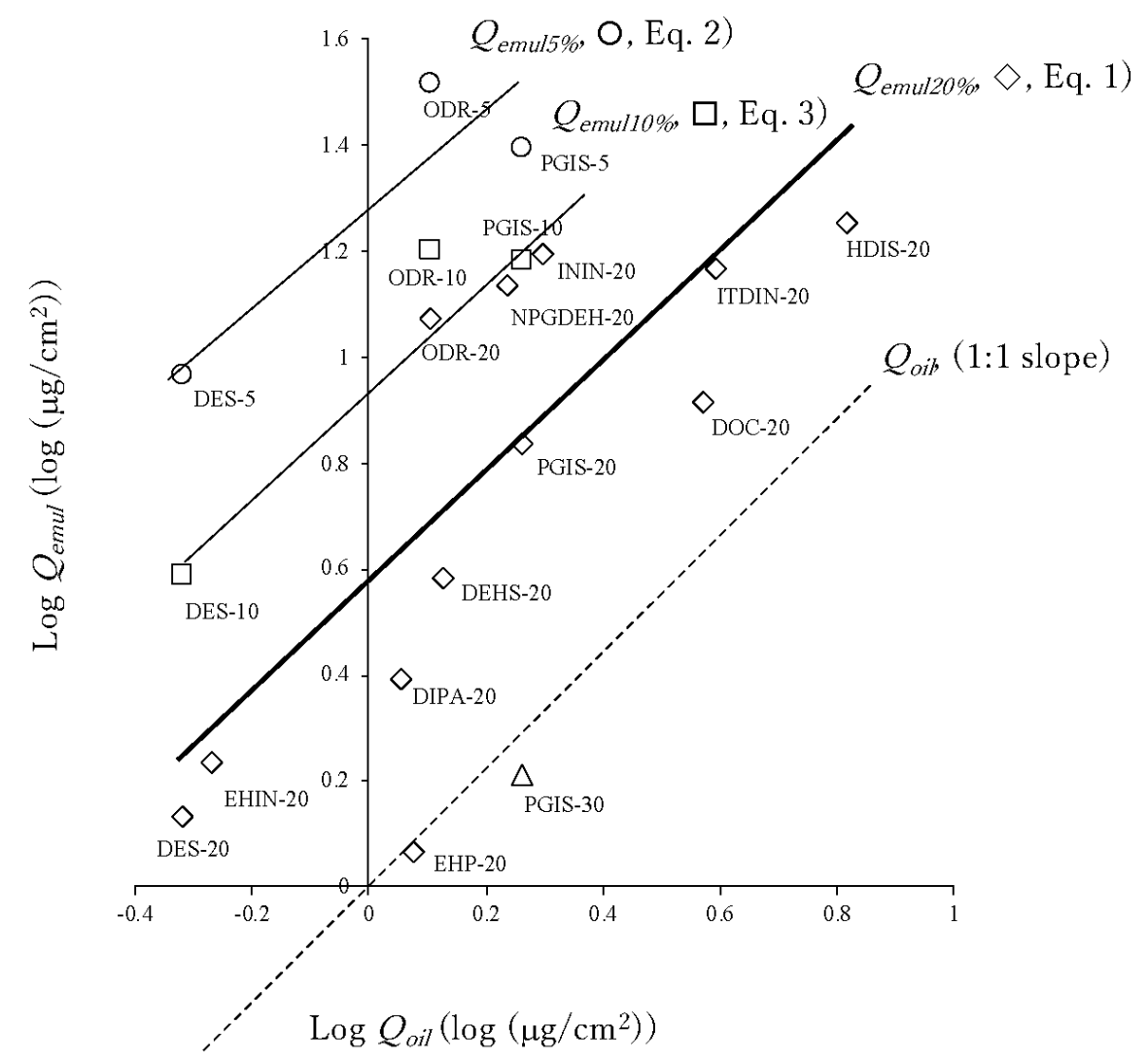

Fig. 4. Prediction of Skin Permeation of FP from $O / W$ Emulsions Containing 5, 10 and $30 \%$ Ester Oil

Lines: Eq. 1) $\log Q_{\text {emul20\% }}=1.04 \times \log Q_{o i l}+0.52\left(R^{2}=0.61\right)$. Eq. 2) $\log Q_{\text {emul } 5^{\circ}}=0.97 \times \log Q_{o j l}+1.25\left(R^{2}=0.80\right)$, Eq. 3) $\log Q_{\text {emull }}=1.11 \times \log Q_{o i l}+0.97\left(R^{2}=0.91\right)$, and Eq. 4) $1: 1$ slope between $\log Q_{\text {oil }}$ and $\log Q_{\text {emul }}$ values. The figure following the ester oil name indicates oil content in the emulsion. Symbols: $\bigcirc$; $5 \%$ ester oil concentration, $\square$; $10 \%$ ester oil concentration, $\diamond ; 20 \%$ ester oil concentration, $\triangle ; 30 \%$ ester oil concentration.

value of compounds from aqueous solution. This linear regression model showed a strong correlation $\left(r^{2}=0.67\right)$ for 93 compounds by the use of $M . W$. and $n$-octanol/water coefficient for individual compounds. ${ }^{1)}$ Furthermore, this model was also modified by multiple descriptors of compounds to cover a wider range of compounds. ${ }^{19-21)}$ In the present study, a multiregression analysis was applied to reveal which molecular descriptors and measured parameters affected the skin permeation of FP from the neat ester oils.

Abraham descriptors have been used to develop in silico skin permeation models in many reports that predict skin permeation of compounds from aqueous solution. ${ }^{22)}$ In the present experiment, measured parameters such as $S C_{\text {oil }}$ and $S_{\text {oil }}$ were involved in addition to molecular descriptors to develop optimal equations. In this model, $S C_{\text {oil }}$ and density acted as positive factors, and $S_{\text {oil }}$ and $\pi_{2}^{H}$ were selected as negatives in the equation for predicting $Q_{\text {oil }}$ value. The thermodynamic activity of drugs shows their escape tendencies from formulations ${ }^{23)}$; therefore factors related to FP solubility in the formulation and in the stratum corneum are reasonable factors in the prediction model. A positive/negative sign of $S C_{o i l}$ and $S_{\text {oil }}$ suggested that drug solvent effect caused by ester oils having a high thermodynamic FP activity provided increased skin permeation. The value of $S C_{\text {oil }}$ might contain the amount adsorbed oil on the stratum corneum, and the amount of adsorbed on the stratum corneum should be changed by characteristic difference of the neat oil. But, in the current method, since it is difficult to distinguish between adsorbed and absorbed oil,
$S C_{\text {oil }}$ was determined by the increased weight of the stratum corneum. Density of ester oil acted as a positive factor. The factor may be selected, because the solvent diffusivity into the stratum corneum affects $S C_{\text {oil }}$ value. However, the concentration/amount of topically applied compounds into the SC at the steady state should be related to its partition coefficient. ${ }^{24)}$ Thus, the $1 \mathrm{~h}$-uptake experiment in the present study may be not enough to reach a steady state for the amount of oil in the stratum corneum.

The amount of ester oil uptake into the SC increased by application of $o / w$ emulsions, and the thermodynamic activity of FP in the emulsions should increase with a decrease of ester oil contents in the formulation due to the lower FP solubility in purified water $\left(S_{\text {water }}, 0.048 \mathrm{mg} / \mathrm{mL}\right.$ at $\left.32^{\circ} \mathrm{C}\right)$. These values and the obtained equation for predicting the $Q_{\text {oil }}$ value may explain a reason for the increased FP permeation by application of $o / w$ emulsions.

A good relationship $\left(R^{2}=0.61\right)$ with a slope of 1.04 was obtained for $Q_{\text {emul20\% }} / Q_{\text {oil }}$, and the relationships between $Q_{\text {emul5\% }} / Q_{\text {oil }}$ or $Q_{\text {emul10\% }} / Q_{\text {oil }}$ were expressed with liner equations having a slope of 0.97 (Eq. 2) and 1.11 (Eq. 3), respectively. These results suggest that the $Q_{\text {emul5\% }}$ and $Q_{\text {emul10\% }}$ values could be expressed by the shift in the $y$-axis intercept in Eq. 1. Interestingly, a constant decrease of FP permeation was observed when formulations having different concentrations of ester oil $(5,10,20,30$ and 100\%) were applied. With this constant slope $(-1.01 \pm 0.33$, the mean \pm S.D. $)$, the increase or decrease in the $Q_{\text {emul }}$ value could be theoretically 
a) PGIS

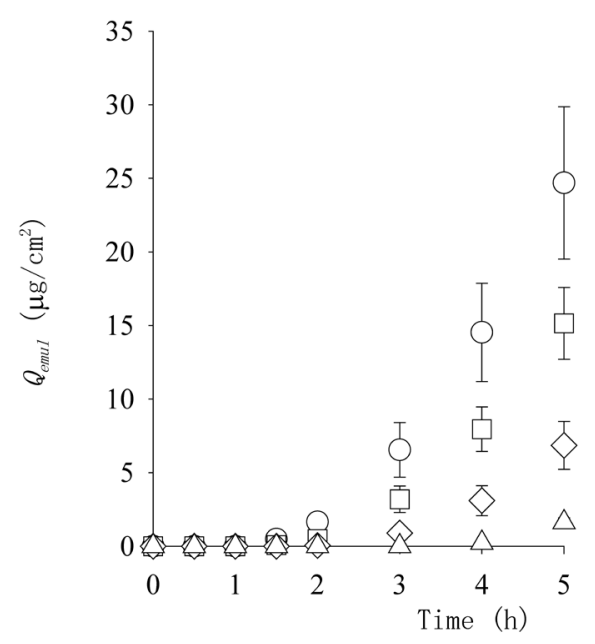

b) $\mathrm{DES}$

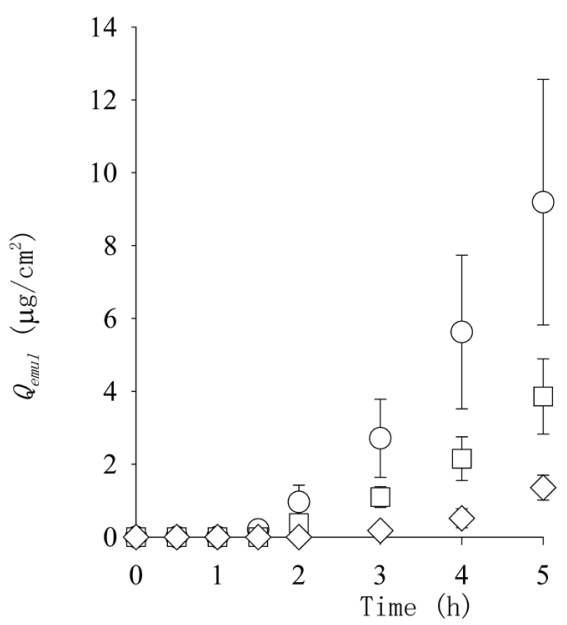

c) ODRA

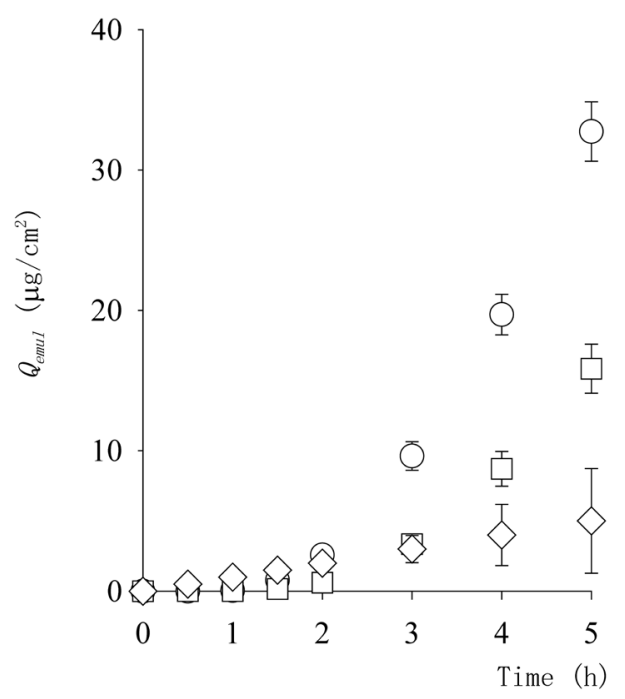

Fig. 5. FP Permeation Profiles through the Skin from $O / W$ Emulsions with Different Concentrations of Ester Oil

Symbols: $\bigcirc ; 5 \%$ ester oil concentration, $\square ; 10 \%$ ester oil concentration, $\diamond ; 20 \%$ ester oil concentration, $\triangle ; 30 \%$ ester oil concentration. a) $o / w$ emulsions composed of PGIS, b) $o / w$ emulsions composed of DES, and c) $o / w$ emulsions composed of ODR. Mean \pm S.E. $(n=3-4)$.

calculated by the use of Eq. 1 as the base equation. The calculated equations were $\log Q_{\text {emul5\% }}=1.04 \times \log Q_{\text {oil }}+1.21$ and $\log$ $Q_{\text {emull } 1 \%}=1.04 \times \log Q_{\text {oil }}+0.83$, respectively. Ester oil concentration in the formulation decides FP solubility in the formulation and the amount of ester oil uptake into the $\mathrm{SC}$, which are the key factors that influence the FP permeation from oil-based formulations. Thus, estimation of $\log Q_{\text {emul5\% }}$ and $\log Q_{\text {emull10\% }}$ values was possible by shifting the $y$-intercept in Eq. 1. In addition, the $\log Q_{\text {emul30\% }}$ value from $o / w$ emulsion consisting of PGIS was almost the same as $\log Q_{\text {oil }}$ value. This result may suggest that $\log Q_{\text {emul }}$ values from ester concentrations of more than $30 \%$ would be equal to $\log Q_{\text {oil }}$ values. As the predictions of $\log Q_{\text {emul } 5 \%}, \log Q_{\text {emullo\% }}$ and $\log Q_{\text {emul } 30 \%}$ were only conducted with several formulations, further experiments are required to confirm that the $y$-intercept shift of Eq. 1. When the prediction method of $Q_{\text {emul20\% }}$ was developed by a regression analysis based on the molecular descriptors and measured parameters, the following equation was obtained;

$$
\begin{aligned}
Q_{\text {emul } 20 \%}= & 30.6( \pm 15.4) S C_{\text {emul }}-202( \pm 78) \pi_{2}^{H} \\
& +0.39( \pm 0.13) M . W .+60.9( \pm 19.9) H A \\
& -2.77( \pm 1.81) I R+1.63( \pm 0.71) S_{\text {emul }} \\
& +149( \pm 59.3) \sum \alpha_{2}^{H} \\
& -203( \pm 64) \sum \beta_{2}^{H}-26.5( \pm 18.9) \\
n=41, R^{2}= & 0.82, F_{0}(8,32)=18.1,(p<0.001)
\end{aligned}
$$

where $S_{\text {emul }}$ is a calculated one by the solubility of in water and in the ester oil $\left(S_{\text {water }}\right.$ and $\left.S_{\text {oil }}\right)$ with the mixing ratio in the emulsion. Instead of the selected factors of $S C_{o i l}$ and $S_{o i l}$ in Eq. 4, $S C_{\text {emul }}$ and $S_{\text {emul }}$ were selected as the factors in Eq. 5 in addition to $M . W ., H A, I R, \Sigma \alpha_{2}{ }^{H}$ and $\Sigma \beta_{2}{ }^{H}$. Nevertheless, $S C_{\text {oil }}$ and $S_{\text {oil }}$ had positive and negative factors, respectively, in Eq. 4 , and both factors of $S C_{e m u l}$ and $S_{\text {emul }}$ had positive factors in Eq. 5. This discrepancy may be due to the difficulty to measure of the accurate $S_{\text {emul }}$ and $S C_{\text {emul }}$. In addition, differences of the particle size and the zeta-potential values of emulsions may also affect the positive/negative signs $(+/-)$ and selected 


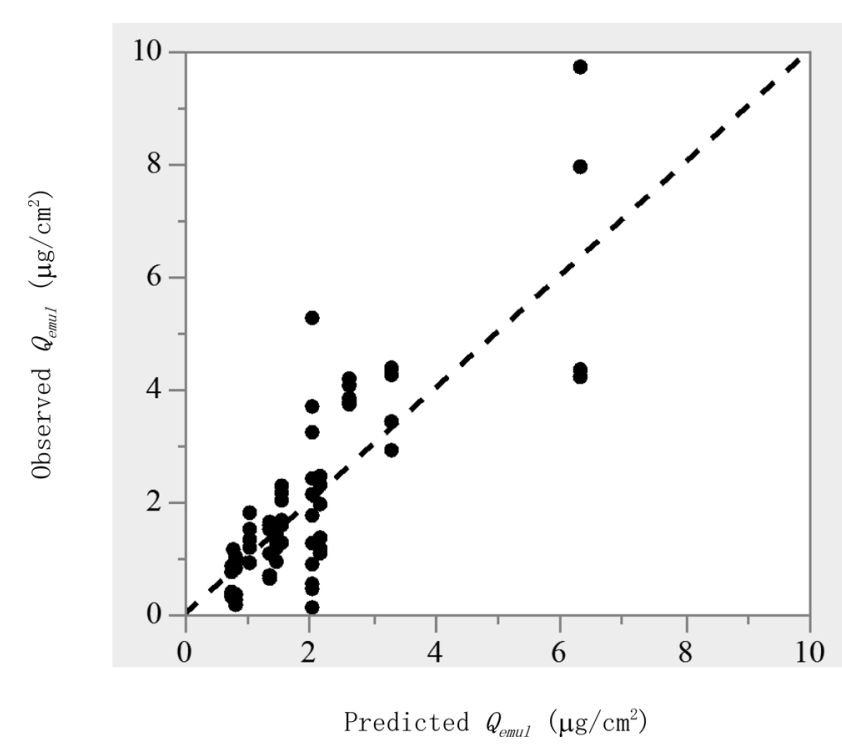

Fig. 6. Four-Fold Cross-Validation Result (Trial \#4) in the Prediction Model for $Q_{\text {oil }}$

RMSE $=1.0316, R_{C V}^{2}=0.62$. The $R_{C V}^{2}$ value denotes $R^{2}$ for each of the crossvalidation result with $Q_{\text {oil }}$ data ( $75 \%$ training and $25 \%$ validation).

factors in Eq. 5 for prediction of $Q_{\text {emul } 20 \%}$ values.

Ester oils such as isopropyl myristate, ${ }^{25)}$ ethyl acetate, ${ }^{26)}$ methyl propionate, ${ }^{27)}$ and butyl acetate ${ }^{28)}$ have been used as skin penetration enhancers. Drug permeation from $o / w$ emulsion consisting of these oils was not evaluated in the present study. As a result, additional or completely different factors that affect the FP permeation may be involved in the optimal equation for predicting $Q_{\text {oil }}$ or $Q_{\text {emul }}$ values.

The present experiment revealed that $S C_{\text {oil }}$ and $S_{\text {oil }}$ were key factors to affect the skin permeation of FP from ester oilbased formulation in addition to molecular descriptors of $\pi_{2}{ }^{H}$ and density. The solubility parameter difference among drug $\left(\delta_{d}\right)$, vehicle $\left(\delta_{v}\right)$ and skin $\left(\delta_{s}\right)$ is well considered in designing dermatological formulations. ${ }^{29,30)}$ There is an inverse relationship between the skin permeation of compounds and the solubility in the vehicle, and the minimum flux and permeability coefficient were observed approximately to the point where $\delta_{d}=\delta_{v}$. In addition, when the $\delta_{d}$ is similar to $\delta_{s}$, which was reported to be approximately $10\left(\mathrm{cal} / \mathrm{cm}^{3}\right)^{1 / 2},{ }^{26)}$ the permeation coefficient of the drug would be increased. In the case of FP application with ester oil, a change of $\delta_{s}$ value toward $\delta_{d}$ may cause the uptake of the ester oil into the stratum corneum. In contrast, the amount of ester oil uptake into the SC may be not a positive factor for an improvement in the skin permeation of hydrophilic drugs. Further experiments are required to verify and expand the results from the present study. For instance, the effect of the polarities of drugs on the selected factors in Eq. 4 and changes in prediction accuracy for skin permeation of drugs from ester oils.

\section{Conclusion}

In the present experiment, the $Q_{\text {emul5-20\% }}$ values were well predicted with the $Q_{\text {oil }}$ values. Furthermore, the optimal equation for prediction of $Q_{\text {oil }}$ was obtained by the measured factors of $S C_{o i l}$ and $S_{o i l}$ and molecular descriptors of $\pi_{2}{ }^{H}$ and density. By the selected factors, topical formulation design like $o / w$ emulsions may be possible considering the skin permeation of drugs. Although more works are necessary to con- firm the applicable range of the obtained equation, the present result may be helpful in deciding a formulation to achieve the desired skin permeation of drugs from $o / w$ emulsions.

Conflict of Interest Kenji Sugibayashi and Hiroaki Todo received a research grant from Kao Corporation.

\section{References}

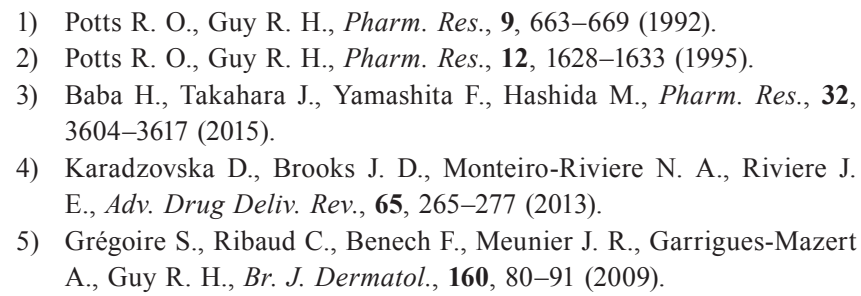

6) Abd E., Yousef S. A., Pastore M. N., Telaprolu K., Mohammed Y. H., Namjoshi S., Grice J. E., Roberts M. S., Clin. Pharmacol. Adv. Appl, 8, 163-176 (2016).

7) Kano S., Todo H., Sugie K., Fujimoto H., Nakada K., Tokudome Y., Hashimoto F., Sugibayashi K., Altern. to Anim. Test. Exp., 15, 61-70 (2010).

8) Uchida T., Kadhum W. R., Kanai S., Todo H., Oshizaka T., Sugibayashi K., Eur. J. Pharm. Sci., 67, 113-118 (2015).

9) Uchida T., Yakumaru M., Nishioka K., Higashi Y., Sano T., Todo H., Sugibayashi K., Chem. Pharm. Bull., 64, 1338-1346 (2016).

10) Sinkó B., Garrigues T. M., Balogh G. T., Nagy Z. K., Tsinman O., Avdeef A., Takács-Novák K., Eur. J. Pharm. Sci., 45, 698-707 (2012).

11) Sloan K. B., Koch S. A. M., Siver K. G., Flowers F. P., J. Invest. Dermatol., 87, 244-252 (1986).

12) Sakata O., Fujii M., Koizumi N., Nakade M., Kameyama K., Watanabe Y., Yakuzaigaku, 74, 84-92 (2014).

13) Roberts M., Cross S., Pellet M., "Dermatological and transdermal formulations", Marcel Dekker, New York, 2002, pp. 89-195.

14) Bouwstra J. A., Gooris G. S., Bras W., Downing D. T., J. Lipid Res., 36, 685-695 (1995).

15) Young J. F., J. Appl. Chem., 17, 241-245 (1967).

16) Uchida T., Nishioka K., Motoki A., Yakumaru M., Sano T., Todo H., Sugibayashi K., Chem. Pharm. Bull., 64, 1597-1606 (2016).

17) Abraham M. H., Martins F., Mitchell R. C., J. Pharm. Pharmacol., 49, 858-865 (1997).

18) Bradley J. C., Abraham M. H., Acree W. E. Jr., Lang A., Chem. Cent. J., 9, 12 (2015).

19) Abraham M. H., Chadha H. S., Mitchell R. C., J. Pharm. Pharmacol., 47, 8-16 (1995).

20) Abraham M. H., Chadha H. S., Martins F., Mitchell R. C., Bradbury M. W., Gratton J. A., Pestic. Sci., 55, 78-88 (1999).

21) Geinoz S., Guy R. H., Testa B., Carrupt P.-A., Pharm. Res., 21, 83-92 (2004).

22) Guy R. H., Hadgraft J., "Drugs and the pharmaceutical sciences," 2nd ed., vol. 123, M. Dekker, New York, 2003.

23) Higuchi T., J. Soc. Cosmet. Chem., 11, 85-93 (1960).

24) Sugibayashi K., Todo H., Oshizaka T., Owada Y., Pharm. Res., 27, 134-142 (2009).

25) Sala M., Diab R., Elaissari A., Fessi H., Int. J. Pharm., 535, 1-17 (2018).

26) Paudel K. S., Milewski M., Swadley C. L., Brogden N. K., Ghosh P., Stinchcomb A. L., Ther. Deliv., 1, 109-131 (2010).

27) Catz P., Friend D. R., Int. J. Pharm., 55, 17-23 (1989).

28) Rosado C., Cross S. E., Pugh W. J., Roberts M. S., Hadgraft J., Pharm. Res., 20, 1502-1507 (2003).

29) Sloan K. B., Siver K. G., Koch A. M., J. Pharm. Sci., 75, 744-749 (1986).

30) Scheler S., Fahr A., Liu X., Admet Dmpk, 2, 199-220 (2015). 